MATHEMATICS OF COMPUTATION

Volume 65, Number 216

October 1996, Pages 1477-1484

\title{
THE BKK ROOT COUNT IN $\mathrm{C}^{n}$
}

\author{
T. Y. LI AND XIAOSHEN WANG
}

\begin{abstract}
The root count developed by Bernshtein, Kushnirenko and Khovanskii only counts the number of isolated zeros of a polynomial system in the algebraic torus $\left(\mathbf{C}^{*}\right)^{n}$. In this paper, we modify this bound slightly so that it counts the number of isolated zeros in $\mathbf{C}^{n}$. Our bound is, apparently, significantly sharper than the recent root counts found by Rojas and in many cases easier to compute. As a consequence of our result, the Huber-Sturmfels homotopy for finding all the isolated zeros of a polynomial system in $\left(\mathbf{C}^{*}\right)^{n}$ can be slightly modified to obtain all the isolated zeros in $\mathbf{C}^{n}$.
\end{abstract}

\section{InTRODUCTION}

A Laurent polynomial $p$ in the $n$ variables $\mathbf{x}=\left(x_{1}, x_{2}, \ldots, x_{n}\right)$ is given by

$$
p(\mathbf{x})=\sum_{\mathbf{e} \in \mathcal{A}} c_{\mathbf{e}} \mathbf{x}^{\mathbf{e}},
$$

where $\mathbf{e}=\left(e_{1}, \ldots, e_{n}\right) \in Z^{n}, \mathbf{x}^{\mathbf{e}}=x_{1}^{e_{1}} \cdots x_{n}^{e_{n}}, c_{\mathbf{e}} \in \mathbf{C}$ and $\mathcal{A}$, the support of $p$, is a finite subset of $Z^{n}$. In short, $p$ is an element of the $\operatorname{ring} \mathbf{C}\left[x_{1}^{ \pm 1}, \ldots, x_{n}^{ \pm 1}\right]$. A Laurent polynomial system $P(\mathbf{x})=\left(p_{1}(\mathbf{x}), \ldots, p_{n}(\mathbf{x})\right)$ is an $n$-tuple of nonzero Laurent polynomials. Let $\mathcal{A}_{i}$ be the support of $p_{i}(\mathbf{x})$, for $i=1, \ldots, n$. Denote the convex hull of $\mathcal{A}_{i}$, called the Newton polytope of $p_{i}$, by $\mathcal{P}_{i}$. Consider the function

$$
R\left(\lambda_{1}, \ldots, \lambda_{n}\right)=\operatorname{Vol}\left(\lambda_{1} \mathcal{P}_{1}+\cdots+\lambda_{n} \mathcal{P}_{n}\right),
$$

where $\lambda_{1}, \ldots, \lambda_{n}$ are nonnegative variables, "Vol" denotes the usual Euclidean volume in $R^{n}$, and

$$
\mathcal{P}_{1}+\cdots+\mathcal{P}_{n}=\left\{\sum_{i=1}^{n} \mathbf{r}_{i}: \quad \mathbf{r}_{i} \in \mathcal{P}_{i} \forall i\right\}
$$

denotes the Minkowski sum of polytopes $\mathcal{P}_{1}, \ldots, \mathcal{P}_{n}$ in $R^{n}$. It is well known in convex geometry [3] that $R\left(\lambda_{1}, \ldots, \lambda_{n}\right)$ is a homogeneous polynomial of degree $n$. The mixed volume $\mathcal{M}\left(\mathcal{A}_{1}, \ldots, \mathcal{A}_{n}\right)$ is defined to be the coefficient of $\lambda_{1} \lambda_{2} \cdots \lambda_{n}$ in this polynomial. Write $\mathbf{C}^{*}$ for the nonzero complex numbers.

Theorem 1.1. The number of isolated zeros, counting multiplicities, of a Laurent polynomial system $P(\mathbf{x})=\left(p_{1}(\mathbf{x}), \ldots, p_{n}(\mathbf{x})\right)$ in $\left(\mathbf{C}^{*}\right)^{n}$ is bounded above by the mixed volume $\mathcal{M}\left(\mathcal{A}_{1}, \ldots, \mathcal{A}_{n}\right)$. For almost all choices of the coefficients of $P(\mathbf{x})$, the number of isolated solutions in $\left(\mathbf{C}^{*}\right)^{n}$ of $P(\mathbf{x})$ is exactly $\mathcal{M}\left(\mathcal{A}_{1}, \ldots, \mathcal{A}_{n}\right)$.

Received by the editor November 23, 1994 and, in revised form, October 9, 1995.

1991 Mathematics Subject Classification. Primary 52B20; Secondary 65H10, 68Q40.

Key words and phrases. BKK bound, mixed volume, homotopy continuation.

The first author's research was supported in part by NSF under Grant DMS-9504953 and by a Guggenheim Fellowship.

(c)1996 American Mathematical Society 
The root count in the above theorem was developed by Bernshtein [1], Kushnirenko [7] and Khovanskii [6], and it is, therefore, commonly referred to as the BKK bound. While this bound is, in general, significantly sharper than the Bézout number (and its variants [11]), it only counts the zeros of $P(\mathbf{x})$ in the $n$-dimensional algebraic torus $\left(\mathbf{C}^{*}\right)^{n}$. In [9], J.M. Rojas proposed a root count formula in $\mathbf{C}^{n}$, derived from the theory of toric varieties, and its computation involves finding the mixed volume of $n$ shadowed polytopes in $R^{n}$.

The main result of this paper can be simply stated as follows:

Given a polynomial system $P(\mathbf{x})=\left(p_{1}(\mathbf{x}), \ldots, p_{n}(\mathbf{x})\right)$ in $\mathbf{C}\left[x_{1}, \ldots, x_{n}\right]$, with support $\mathcal{A}_{i}$ for $p_{i}, i=1, \ldots, n$, the number of isolated zeros, counting multiplicities, of $P(\mathbf{x})$ in $\mathbf{C}^{n}$ is bounded above by the mixed volume $\mathcal{M}\left(\mathcal{A}_{1} \cup\{0\}, \ldots, \mathcal{A}_{n} \cup\{0\}\right)$.

Our bound is significantly tighter than the root count suggested in [9]. A simple explanation derives from the monotonicity of the mixed volume: growing polytopes potentially increases (and never decreases) the mixed volume; and the support sets for which we compute the mixed volumes here are usually much smaller than the shadowed sets used in [9]. Furthermore, our bound appears to be easier to compute. This bound also holds over arbitrary algebraically closed fields [10].

Of course, if $\mathcal{A}_{i} \cup\{0\}=\mathcal{A}_{i}$ for all $i=1, \ldots, n$ (i.e., if each $p_{i}$ already has a constant term), then, by Theorem 1.1, our bound is exact when the coefficients of the $p_{i}$ 's are chosen generically. When $0 \notin \mathcal{A}_{i}$ for some $i$, we will show in $\S 2$ that our bound is exact when the coefficients of the $p_{i}$ 's are chosen generically and the zeros of $P(\mathbf{x})$ at infinity are nonsingular. An alternative combinatorial criterion to guarantee the exactness of this bound is given in [10]. In contrast to the complex analytic approach given in this paper, the method in [10] is more algebraic and the conditions are formulated in a more general setting.

The idea of the BKK bound has been used in several different ways in homotopy continuation methods for solving polynomial systems $[5,12,13]$. The lifting homotopy approach in [5] is particularly attractive since only a small number of homotopy curves need to be followed. Nonetheless, as was pointed out in that paper, an apparent limitation of the method is that it finds zeros of the polynomial system only in $\left(\mathbf{C}^{*}\right)^{n}$, and not necessarily all zeros in the affine space $\mathbf{C}^{n}$. As a consequence of our result, we shall show in $\S 3$ that the lifting homotopy approach in [5] can be easily modified to obtain all the isolated zeros of a polynomial system in $\mathbf{C}^{n}$.

\section{MAIN RESUlts}

Complex $n$-space $\mathbf{C}^{n}$ can be naturally embedded in the $n$-dimensional manifold

$$
\mathbf{P}^{n}=\left\{\mathbf{x}=\left(x_{0}, \ldots, x_{n}\right) \in \mathbf{C}^{n+1}: \mathbf{x} \neq(0, \ldots, 0)\right\} / \sim,
$$

where the equivalence relation $\sim$ is given by $\mathbf{x} \sim \mathbf{y}$ if and only if $\mathbf{x}=c \mathbf{y}$ for some nonzero $c \in \mathbf{C}$. The original $\mathbf{C}^{n}$ corresponds to points $\mathbf{x}=\left(x_{0}, \ldots, x_{n}\right)$ with $x_{0}=1$, and $x_{0}=0$ defines the projective hyperplane of points at infinity. Thus, $\mathbf{P}^{n}$ is the disjoint union of $\mathbf{C}^{n}$ and the points at infinity.

Given a polynomial $p(\mathbf{x})$ of degree $d$ in the $n$ variables $x_{1}, \ldots, x_{n}$, define $\bar{p}\left(x_{1}, \ldots, x_{n}\right)$ to be the homogeneous polynomial consisting of all the degree- $d$ terms of $p$, and define the homogenization of $p$ to be

$$
\tilde{p}\left(x_{0}, \ldots, x_{n}\right)=x_{0}^{d} p\left(\frac{x_{1}}{x_{0}}, \ldots, \frac{x_{n}}{x_{0}}\right) .
$$


Note that $\tilde{p}$ is a homogeneous polynomial of degree $d$ in the $n+1$ variables $x_{0}, \ldots, x_{n}$, and $\tilde{p}\left(1, x_{1}, \ldots, x_{n}\right)=p\left(x_{1}, \ldots, x_{n}\right)$ and $\tilde{p}\left(0, x_{1}, \ldots, x_{n}\right)=\bar{p}\left(x_{1}, \ldots, x_{n}\right)$. Geometrically speaking, we can define the zero set in $\mathbf{P}^{n}$ of the system $\tilde{P}=\left(\tilde{p}_{1}, \ldots, \tilde{p}_{n}\right)$ via the disjoint union

$$
X=\left\{\left(1, x_{1}, \ldots, x_{n}\right) \mid P\left(x_{1}, \ldots, x_{n}\right)=0\right\} \cup\left\{\left(0, x_{1}, \ldots, x_{n}\right) \mid \bar{P}\left(x_{1}, \ldots, x_{n}\right)=0\right\} .
$$

Thus, $X$ is the union of the zeros of $P$ in $\mathbf{C}^{n}$ and the zeros at infinity.

A projective variety is the zero set in $\mathbf{P}^{n}$ of a set $\left\{f_{1}, \ldots, f_{r}\right\}$ of homogeneous polynomials in the variables $x_{0}, \ldots, x_{n}$. A quasi-projective variety is the complement $Y_{1} \backslash Y_{2}$ of a projective subvariety $Y_{2}$ in another projective variety $Y_{1}$. Since $Y_{2}$ may be the empty set, quasi-projective varieties are generalizations of projective varieties. The zero set of a finite number of polynomials in $\mathbf{C}^{n}$ is a quasi-projective variety, but not necessarily a projective variety.

Suppose $Y$ is the zero set in $\mathbf{P}^{n}$ of a set of homogeneous polynomials $\left\{f_{1}, \ldots, f_{r}\right\}$. A point $\mathbf{y} \in Y$ is nonsingular (or regular) if and only if

$$
\operatorname{rank}_{C} \frac{\partial\left(f_{1}, \ldots, f_{r}\right)}{\partial\left(x_{0}, \ldots, x_{n}\right)}(\mathbf{y})=\operatorname{codim} Y,
$$

where codim $Y=n-\operatorname{dim}_{\mathbf{P}^{n}} Y$. A variety is nonsingular if and only if each point of the variety is nonsingular.

The classical Bertini theorem implies that nonsingularity is a generic property. A linear system $L$ is a complex vector space generated by homogeneous polynomials of some fixed degree, i.e.,

$$
L=\left\{\sum_{i=1}^{r} c_{i} h_{i} \mid\left(c_{1}, \ldots, c_{r}\right) \in \mathbf{C}^{r}\right\}
$$

for $h_{1}, \ldots, h_{r} \in \mathbf{C}\left[x_{0}, \ldots, x_{n}\right]$. The base locus of $L$ is the set

$$
\left\{\mathbf{x} \in \mathbf{P}^{n} \mid h_{i}(\mathbf{x})=0, \quad i=0, \ldots, r\right\} .
$$

Bertini's Theorem ([3, Ch. III, $\S 10.9 .2])$. Let $Y$ be a nonsingular quasi-projective subvariety of $\mathbf{P}^{n}$ of dimension $m$. Let

$$
L=\left\{\sum_{i=1}^{r} c_{i} h_{i} \mid\left(c_{1}, \ldots, c_{r}\right) \in \mathbf{C}^{r}\right\}
$$

be a linear system parametrized by $\mathbf{C}^{r}$. Then there exists a nonzero homogeneous polynomial $G$ in $r$ variables such that $G\left(c_{1}, \ldots, c_{r}\right) \neq 0$ implies that the intersection of $Y$ with the hypersurface

$$
\sum_{i=1}^{r} c_{i} h_{i}=0
$$

is of dimension $m-1$ and is nonsingular at each point not in the base locus of $L$.

Lemma 2.1. Given a polynomial system $P(\mathbf{x})=\left(p_{1}(\mathbf{x}), \ldots, p_{n}(\mathbf{x})\right)$ in the variables $\mathbf{x}=\left(x_{1}, \ldots, x_{n}\right)$, there exists an open dense subset $V$ of $\mathbf{C}^{n}$ such that if 
$\varepsilon=\left(\varepsilon_{1}, \ldots, \varepsilon_{n}\right) \in V$, then $t \varepsilon \in V$ for all $t \neq 0$ in $R^{1}$, and if $\mathbf{y} \in \mathbf{C}^{n}$ is a solution of

$$
\begin{aligned}
q_{1} & =p_{1}\left(x_{1}, \ldots, x_{n}\right)+\varepsilon_{1}=0, \\
& \cdot \\
& \cdot \\
& \cdot \\
q_{n} & =p_{n}\left(x_{1}, \ldots, x_{n}\right)+\varepsilon_{n}=0,
\end{aligned}
$$

then

$$
\operatorname{rank}_{C} \frac{\partial\left(q_{1}, \ldots, q_{n}\right)}{\partial\left(x_{1}, \ldots, x_{n}\right)}(\mathbf{y})=n \quad \text { and }
$$

where $\mathbf{C}^{*}=\mathbf{C} \backslash\{0\}$.

$$
\mathbf{y} \in\left(\mathbf{C}^{*}\right)^{n},
$$

Proof. (i) For $i=1, \ldots, n$, let $d_{i}$ be the degree of $p_{i}$. Write the homogenizations of the polynomials in (1) as

$$
\begin{aligned}
\tilde{q}_{1} & =\tilde{p}_{1}\left(x_{0}, \ldots, x_{n}\right)+\varepsilon_{1} x_{0}^{d_{1}}, \\
& \cdot \\
& \cdot \\
& \cdot \\
\tilde{q}_{n} & =\tilde{p}_{n}\left(x_{0}, \ldots, x_{n}\right)+\varepsilon_{n} x_{0}^{d_{n}} .
\end{aligned}
$$

Consider the homogeneous polynomials

$$
\begin{aligned}
f_{1} & =a_{1} \tilde{p}_{1}\left(x_{0}, \ldots, x_{n}\right)+\varepsilon_{1} x_{0}^{d_{1}}, \\
& \cdot \\
& \cdot \\
& \cdot \\
f_{n} & =a_{n} \tilde{p}_{n}\left(x_{0}, \ldots, x_{n}\right)+\varepsilon_{n} x_{0}^{d_{n}},
\end{aligned}
$$

for $a_{i} \in \mathbf{C}, i=1, \ldots, n$. Let $Y_{0}=\mathbf{P}^{n} \backslash\left\{x_{0}=0\right\}=\mathbf{C}^{n}$. $Y_{0}$ is a quasi-projective subvariety of $\mathbf{P}^{n}$. Consider the linear system $\left\{f_{1}\right\}$ formed by $x_{0}^{d_{1}}$ and $\tilde{p}_{1}\left(x_{0}, \ldots, x_{n}\right)$ as $\left(a_{1}, \varepsilon_{1}\right)$ vary in $\mathbf{C}^{2}$. The base locus of the linear system is contained in $\left\{x_{0}=0\right\}$. Therefore, by Bertini's theorem, there exists a nonzero homogeneous polynomial $G_{1}\left(a_{1}, \varepsilon_{1}\right)$ such that the intersection $Y_{0} \cap\left\{f_{1}=0\right\}$ is a nonsingular quasi-projective variety for $G_{1}\left(a_{1}, \varepsilon_{1}\right) \neq 0$. Since $G_{1}$ is homogeneous, the polynomial $H_{1}\left(\varepsilon_{1}\right)=$ $G_{1}\left(1, \varepsilon_{1}\right)$ is not identically zero, and therefore vanishes on a subvariety $T_{1}$ of $\mathbf{C}$. Thus, $T_{1}$ can have only finitely many points, so $T_{1}^{0}=\left\{t T_{1}: t \in R^{1} \backslash\{0\}\right\}$ is of one real dimension in $\mathbf{C}$, and its complement $V_{1}$ in $\mathbf{C}$ is open and dense in $\mathbf{C}$. The set $V_{1}$ has the property that if $\varepsilon_{1} \in V_{1}$, then $t \varepsilon_{1} \in V_{1}$ for real $t \neq 0$, and $Y_{1}\left(\varepsilon_{1}\right)=Y_{0} \cap\left\{\tilde{q}_{1}=0\right\}$ is nonsingular and is of dimension $n-1$.

Next, we repeat this process $n-1$ times to construct $Y_{2}, \ldots, Y_{n}$ by $Y_{k}\left(\varepsilon_{1}, \ldots, \varepsilon_{k}\right)=$ $Y_{k-1}\left(\varepsilon_{1}, \ldots, \varepsilon_{k-1}\right) \cap\left\{\tilde{q}_{k}=0\right\}$. Here, $Y_{k-1}$ is a quasi-projective subvariety of $\mathbf{P}^{n}$ of complex dimension $n-k+1$. The base locus of the linear system $\left\{f_{k}\right\}$, formed by $x_{0}^{d_{k}}$ and $\tilde{p}_{k}\left(x_{0}, \ldots, x_{n}\right)$ as $\left(a_{k}, \varepsilon_{k}\right)$ vary in $\mathbf{C}^{2}$, is contained in $\left\{x_{0}=0\right\}$. Hence, it does not intersect $Y_{k}$. Applying Bertini's theorem, for all $\left(\varepsilon_{1}, \ldots, \varepsilon_{k-1}\right) \in V_{k-1}$ there exists 
an open dense subset $V_{k}^{\prime}\left(\varepsilon_{1}, \ldots, \varepsilon_{k-1}\right)$ of $\mathbf{C}$ such that for $\varepsilon_{k} \in V_{k}^{\prime}$, we have $t \varepsilon_{k} \in V_{k}^{\prime}$ for real $t \neq 0$, and $Y_{k}$ is of dimension $n-k$ and is nonsingular. We can then easily show that the set $V_{k}=\left\{\left(\varepsilon_{1}, \ldots, \varepsilon_{k}\right) \mid\left(\varepsilon_{1}, \ldots, \varepsilon_{k-1}\right) \in V_{k-1}, \varepsilon_{k} \in V_{k}^{\prime}\left(\varepsilon_{1}, \ldots, \varepsilon_{k-1}\right)\right\}$ is open dense, and invariant under nonzero real scalings.

We then let $V^{1}=V_{n}$ and note that $V^{1}$ is an open dense set in $\mathbf{C}^{n}$ and for $\varepsilon=\left(\varepsilon_{1}, \ldots, \varepsilon_{n}\right) \in V^{1}$, we have $t \varepsilon \in V^{1}$ for real $t \neq 0$, and the finite solution set of (1) is of zero dimension and nonsingular. The conclusion follows.

(ii) We will show that for each $i=1, \ldots, n$, there exists an open dense set $U_{i} \subset \mathbf{C}^{n}$ such that for $\varepsilon=\left(\varepsilon_{1}, \ldots, \varepsilon_{n}\right) \in U_{i}$, te $\in U_{i}$ for real $t \neq 0$ and the polynomial equations

$$
\begin{array}{ccc}
q_{1}= & p_{1}\left(x_{1}, \ldots, x_{n}\right)+\varepsilon_{1} & =0 \\
& \vdots & \\
q_{n}= & p_{n}\left(x_{1}, \ldots, x_{n}\right)+\varepsilon_{n} & =0 \\
q_{n+1}= & x_{i} & =0
\end{array}
$$

have no common solutions. Then the conclusion follows if we let

$$
V^{2}=\bigcap_{i=1}^{n} U_{i}
$$

The system in (4) is equivalent to the following system of $n$ equations in the $n-1$ variables $x_{1}, \ldots, x_{i-1}, x_{i+1}, \ldots, x_{n}$ :

$$
p_{1}\left(x_{1}, \ldots x_{i-1}, 0, x_{i+1}, \ldots, x_{n}\right)+\varepsilon_{1}=0,
$$

$$
p_{n}\left(x_{1}, \ldots x_{i-1}, 0, x_{i+1}, \ldots, x_{n}\right)+\varepsilon_{n}=0 .
$$

Denote the system $(5)$ by $Q(x, \varepsilon)$. Following the same procedure as in (i), we obtain an open dense subset $U_{i}$ in $\mathbf{C}^{n}$ such that for $\varepsilon=\left(\varepsilon_{1}, \ldots, \varepsilon_{n}\right) \in U_{i}$, we have $t \varepsilon \in U_{i}$ for real $t \neq 0$, and for any solution $\mathbf{y}$ of (5), the rank of $D_{x} Q$ is $n$. But this is impossible. So, the solution set of (5) is empty.

To accommodate both (i) and (ii), we may let $V=V^{1} \cap V^{2}$. Then for $\varepsilon=$ $\left(\varepsilon_{1}, \ldots, \varepsilon_{n}\right) \in V$, we have $t \varepsilon \in V$ for real $t \neq 0$, and the assertions in both (i) and (ii) follow.

Lemma 2.2. Given a polynomial system $P(\mathbf{x})=\left(p_{1}(\mathbf{x}), \ldots, p_{n}(\mathbf{x})\right)$ in the variables $\mathbf{x}=\left(x_{1}, \ldots, x_{n}\right)$, let $k_{\varepsilon}$ and $k$ be the numbers of isolated zeros, counting multiplicities, in $\mathbf{C}^{n}$ of $P_{\varepsilon}(\mathbf{x})=\left(p_{1}(\mathbf{x})+\varepsilon_{1}, \ldots, p_{n}(\mathbf{x})+\varepsilon_{n}\right)$ and $P(\mathbf{x})$, respectively. Then,

$$
k_{\varepsilon} \geq k
$$

for small $\varepsilon_{1}, \ldots, \varepsilon_{n}$.

Proof. Let $x_{0}$ be an isolated zero of $P(\mathbf{x})$ with multiplicity $m \geq 1$. Let $N$ be an open neighborhood of $x_{0}$ containing no other solution of $P(\mathbf{x})=0 . \operatorname{Let} \operatorname{deg}(P, N, 0)$ be the Brouwer degree (see [2]), where $P(\mathbf{x})$ is regarded as a function on $R^{2 n}$, the space induced by $\mathbf{C}^{n}$ in a natural fashion. In [2] it is $\operatorname{shown}$ that $\operatorname{deg}(P, N, 0)$ is always a positive integer and equal to $m$. Since the Brouwer degree is invariant under small perturbations, for small $\varepsilon$, we have $\operatorname{deg}\left(P_{\varepsilon}, N, 0\right)=m$, where $P_{\varepsilon}$ is 
regarded as a function on $R^{2 n}$. Therefore, the number of isolated zeros $k_{\varepsilon}$ of $P_{\varepsilon}$ is at least as big as the number of isolated zeros $k$, counting multiplicities, of $P(\mathbf{x})$.

Example 1. The system

$$
\begin{aligned}
x_{2} x_{1}^{2}-x_{1}+1 & =0 \\
x_{2} & =0
\end{aligned}
$$

has only one solution, $\left(x_{1}, x_{2}\right)=(1,0)$. However, its constant-term perturbation system

$$
\begin{array}{r}
x_{2} x_{1}^{2}-x_{1}+1+\varepsilon_{1}=0 \\
x_{2}+\varepsilon_{2}=0
\end{array}
$$

has two isolated solutions, namely,

$$
\left(x_{1}, x_{2}\right)=\left(\frac{-1 \pm \sqrt{1+4 \varepsilon_{2}\left(1+\varepsilon_{1}\right)}}{2 \varepsilon_{2}},-\varepsilon_{2}\right) .
$$

The following proposition gives a sufficient condition that can be easily verified when equality in (6) holds. Recall that for a polynomial system $P\left(x_{1}, \ldots, x_{n}\right)=$ $\left(p_{1}\left(x_{1}, \ldots, x_{n}\right), \ldots, p_{n}\left(x_{1}, \ldots, x_{n}\right)\right)$, the zero set at infinity of $P$ is the set $\left\{\left(0, x_{1}, \ldots, x_{n}\right)\right.$ $\left.\in \mathbf{P}^{n} \mid \bar{P}\left(x_{1}, \ldots, x_{n}\right)=0\right\}$, where $\bar{P}\left(x_{1}, \ldots, x_{n}\right)=\left(\bar{p}_{1}\left(x_{1}, \ldots, x_{n}\right), \ldots, \bar{p}_{n}\left(x_{1}, \ldots, x_{n}\right)\right)$ and each $\bar{p}_{i}\left(x_{1}, \ldots, x_{n}\right)$ is a homogeneous polynomial consisting of the highest-degree terms of $p_{i}\left(x_{1}, \ldots, x_{n}\right)$.

Proposition 2.3. Equality in (6) holds if the zero set at infinity of $P(\mathbf{x})=$ $\left(p_{1}(\mathbf{x}), \ldots, p_{n}(\mathbf{x})\right)$ is nonsingular.

Proof. Clearly, the zero set at infinity of $P_{\varepsilon}(\mathbf{x})=\left(p_{1}(\mathbf{x})+\varepsilon_{1}, \ldots, p_{n}(\mathbf{x})+\varepsilon_{n}\right)$, denoted by $S_{0}$, is the same as that of $P(\mathbf{x})$, denoted by $S_{1}$. If $S_{1}$ is nonsingular, since small perturbations do not change the rank of the appropriate Jacobian, $S_{0}$ must also be nonsingular. On the other hand, from (i) in Lemma 2.1, the zero set of $P_{\varepsilon}$ in $\mathbf{C}^{n}$ consists of nonsingular isolated points for generic $\varepsilon=\left(\varepsilon_{1}, \ldots, \varepsilon_{n}\right)$, namely, if $\mathbf{w} \in \mathbf{C}^{n}$ and $P_{\varepsilon}(\mathbf{w})=0$, then

$$
\operatorname{rank}_{C} \frac{\partial P_{\varepsilon}\left(x_{1}, \ldots, x_{n}\right)}{\partial\left(x_{1}, \ldots, x_{n}\right)}(\mathbf{w})=n .
$$

Now consider the homotopy $H: \mathbf{C}^{n} \times[0,1] \rightarrow \mathbf{C}^{n}$ defined by

$$
H(x, t)=(1-t) P_{\varepsilon}(\mathbf{x})+t P(\mathbf{x})=0 .
$$

It was shown in [8] that when the zero set at infinity of $P_{\varepsilon}(\mathbf{x})$ is nonsingular and equals that of $P(\mathbf{x})$, which is also nonsingular, then any homotopy curve $\mathbf{x}(t)$ emanating from an isolated zero of $P_{\varepsilon}(\mathbf{x})$ at $t=0$ always connects to an isolated zero of $P(\mathbf{x})$ when $t \rightarrow 1$. From this, the conclusion follows.

Theorem 2.4. For a polynomial system $P(\mathbf{x})=\left(p_{1}(\mathbf{x}), \ldots, p_{n}(\mathbf{x})\right)$, let $\mathcal{A}_{1}, \ldots, \mathcal{A}_{n}$ be the supports of $p_{1}(\mathbf{x}), \ldots, p_{n}(\mathbf{x})$, respectively. Then, the mixed volume $\mathcal{M}\left(\mathcal{A}_{1} \cup\{0\}, \ldots, \mathcal{A}_{n} \cup\{0\}\right)$ is an upper bound for the number of isolated zeros, counting multiplicities, of $P(\mathbf{x})=\left(p_{1}(\mathbf{x}), \ldots, p_{n}(\mathbf{x})\right)$ in $\mathbf{C}^{n}$. 
Proof. For $P_{\varepsilon}(\mathbf{x})=\left(p_{1}(\mathbf{x})+\varepsilon_{1}, \ldots, p_{n}(\mathbf{x})+\varepsilon_{n}\right), \mathcal{A}_{1} \cup\{0\}, \ldots, \mathcal{A}_{n} \cup\{0\}$ are the supports of $p_{1}(\mathbf{x})+\varepsilon_{1}, \ldots, p_{n}(\mathbf{x})+\varepsilon_{n}$, respectively. Thus,

$$
\begin{aligned}
& \mathcal{M}\left(\mathcal{A}_{1} \cup\{0\}, \ldots, \mathcal{A}_{n} \cup\{0\}\right) \geq \text { the number of isolated zeros } \\
& \text { of } P_{\varepsilon}(\mathbf{x}) \text { in }\left(\mathbf{C}^{*}\right)^{n} \quad \text { ( by Theorem 1.1) } \\
&= \text { the number of isolated zeros of } P_{\varepsilon}(\mathbf{x}) \text { in } \mathbf{C}^{n} \quad \text { ( by Lemma 2.1) } \\
& \geq \text { the number of isolated zeros of } P(\mathbf{x}) \text { in } \mathbf{C}^{n} \quad \text { ( by Lemma 2.2). }
\end{aligned}
$$

Corollary 2.5. In the theorem above, fix $\mathcal{A}_{1}, \ldots, \mathcal{A}_{n}$ and now suppose that the support of $p_{i}$ is contained in $\mathcal{A}_{i}$, for $i=1, \ldots, n$. Then

(i) If $0 \in \mathcal{A}_{i}$ for all $i=1, \ldots, n$, that is, each $p_{i}(\mathbf{x})$ has a constant term, then the bound is exact when the coefficients of $P(\mathbf{x})$ are chosen generically.

(ii) Otherwise, if the zero set at infinity of $P(\mathbf{x})$ is nonsingular for generically chosen coefficients, then the bound is exact when the coefficients of $P(\mathbf{x})$ are chosen generically.

Proof. Part (i) is a direct consequence of Theorem 1.1. Part (ii) follows from Proposition 2.3 and Theorem 1.1.

\section{The Huber-Sturmfels homotopy}

One of the major steps in the homotopy continuation approach to solving for all the isolated zeros in $\left(\mathbf{C}^{*}\right)^{n}$ of a polynomial system $P(\mathbf{x})=\left(p_{1}(\mathbf{x}), \ldots, p_{n}(\mathbf{x})\right)$, $\mathbf{x}=\left(x_{1}, \ldots, x_{n}\right)$, suggested by Huber and Sturmfels in [5], can be described as follows.

Let $Q(\mathbf{x})$ be a polynomial system with the same support set as $P(\mathbf{x})$ but with sufficiently randomly chosen complex coefficients. By Theorem 1.1, $Q(\mathbf{x})$ has as many isolated zeros in $\left(\mathbf{C}^{*}\right)^{n}$ as its BKK bound, say $k$ zeros. Now, consider the homotopy

$$
\begin{aligned}
H(\mathbf{x}, t) & =(1-t) Q(\mathbf{x})+t P(\mathbf{x}) \\
& =Q(\mathbf{x})+t(P(\mathbf{x})-Q(\mathbf{x})), \quad t \in[0,1] .
\end{aligned}
$$

For each $t \in[0,1]$, the support sets of $H(\mathbf{x}, t)$ are the same as those of $Q(\mathbf{x})$ and the coefficients are generic, so for each $t \in[0,1]$ the number of isolated zeros of $H(\mathbf{x}, t)$ in $\left(\mathbf{C}^{*}\right)^{n}$ is equal to $k$. Accordingly, each isolated zero of $P(\mathbf{x})$ in $\left(\mathbf{C}^{*}\right)^{n}$ can be reached by a homotopy curve of $H(\mathbf{x}, t)=0$, emanating from an isolated zero of $Q(\mathbf{x})$ in $\left(\mathbf{C}^{*}\right)^{n}$ at $t=0$.

In [5], Huber and Sturmfels proposed using the lifting homotopy on $H(\mathbf{x}, t)$ in (7) to define another homotopy $\mathcal{H}(\mathbf{x}, t)$ (with the same $t$ ) with $\mathcal{H}(\mathbf{x}, 1)=P(\mathbf{x})$. Then the solutions of the lowest-order terms of the Puiseux series of the solution branches of $\mathcal{H}(\mathbf{x}, t)=0$ are used as the starting points to follow the homotopy curves of $\mathcal{H}(\mathbf{x}, t)=0$.

This approach is very promising, since only a small number of homotopy curves need to be followed. However, as mentioned in [5], an apparent limitation of their method is that it finds only the zeros in $\left(\mathbf{C}^{*}\right)^{n}$.

In fact, in order to find all the isolated zeros of $P(\mathbf{x})$ in $\mathbf{C}^{n}$ rather than in $\left(\mathbf{C}^{*}\right)^{n}$, one needs only to modify the homotopy in (7) slightly by replacing $Q(\mathbf{x})$ with

$$
Q_{\varepsilon}(\mathbf{x})=Q(\mathbf{x})+\varepsilon,
$$

where $\varepsilon=\left(\varepsilon_{1}, \ldots, \varepsilon_{n}\right)$ is randomly chosen in $\mathbf{C}^{n}$. First of all, by Lemma 2.1, all zeros of $Q_{\varepsilon}(\mathbf{x})$ are isolated, nonsingular and in $\left(\mathbf{C}^{*}\right)^{n}$. Therefore, the BKK bound 
$k_{\varepsilon}$ of $Q_{\varepsilon}(\mathbf{x})$ equals the number of isolated zeros in $\mathbf{C}^{n}$ of $Q_{\varepsilon}(\mathbf{x})$. Secondly, for each $t_{0} \in[0,1)$, the support of

$$
\begin{aligned}
H_{\varepsilon}\left(\mathbf{x}, t_{0}\right) & =\left(1-t_{0}\right) Q_{\varepsilon}(\mathbf{x})+t_{0} P(\mathbf{x}) \\
& =Q_{\varepsilon}(\mathbf{x})+t_{0}\left(P(\mathbf{x})-Q_{\varepsilon}(\mathbf{x})\right) \\
& =Q(\mathbf{x})+t_{0}(P(\mathbf{x})-Q(\mathbf{x}))+\left(1-t_{0}\right) \varepsilon
\end{aligned}
$$

is the same as that of $Q_{\varepsilon}(\mathbf{x})$, and by Lemma 2.2, the set of all the isolated zeros of $H_{\varepsilon}\left(\mathbf{x}, t_{0}\right)$ in $\mathbf{C}^{n}$ is contained in $\left(\mathbf{C}^{*}\right)^{n}$ and its cardinality must be equal to $k_{\varepsilon}$. Consequently, there are exactly $k_{\varepsilon}$ homotopy curves of $H_{\varepsilon}(\mathbf{x}, t)=0$ in $\mathbf{C}^{n} \times[0,1]$, and any homotopy curve $\mathbf{x}(t)$ of $H_{\varepsilon}(\mathbf{x}, t)=0$ with $\mathbf{x}(1)$ being a zero of $P(\mathbf{x})$, either in $\left(\mathbf{C}^{*}\right)^{n}$ or in $\mathbf{C}^{n}$, must join one of those $k_{\varepsilon}$ curves.

\section{REFERENCES}

1. D. N. Bernshtein, The number of roots of a system of equations, Functional Anal. Appl., 9(3): 183-185, 1975. MR 55:8034

2. F. E. Browder, Nonlinear mappings of analytic type in Banach spaces, Math. Ann., 185:259278, 1970. MR 41:4318

3. T. Bonnesen and W. Fenchel, Theory of convex bodies, BCS Associates, Moscow, Idaho, 1987. MR 88j:52001

4. R. Hartshorne, Algebraic geometry, Graduate Texts in Math., 52, Springer-Verlag, Berlin, Heidelberg, New York, 1977. MR 57:3116

5. B. Huber and B. Sturmfels, A polyhedral method for solving sparse polynomial systems, Math. Comp., 64: 1541-1555, 1995. MR 95m:65100

6. A. G. Khovanskii, Newton polyhedra and the genus of complete intersections, Functional Anal. Appl., 12(1):38-46, 1978. MR 80b:14022

7. A. G. Kushnirenko, Newton polytopes and the Bézout theorem, Functional Anal. Appl., 10(3):233-235, 1976. MR 54:10263

8. T. Y. Li, T. Sauer and J.A. Yorke, The random product homotopy and deficient polynomial systems, Numer. Math., 51:481-500, 1987. MR 88j:65108

9. J. M. Rojas, A convex geometric approach to counting the roots of a polynomial system, Theoret. Comput. Sci. 133 (1994), 105-140. MR 95k:68090

10. J. M. Rojas and X. Wang, Counting affine roots via pointed Newton polytopes, to appear: J. of Complexity.

11. I. R. Shafarevich, Basic algebraic geometry, Springer, New York, 1977. MR 56:5538

12. J. Verschelde and R. Cools, Symbolic homotopy construction, Applicable Algebra in Engineering, Communication and Computing, 4(3):169-183, 1993. MR 94c:68102

13. J. Verschelde, P. Verlinden and R. Cools, Homotopies exploiting Newton polytopes for solving sparse polynomial systems, SIAM J. Numer. Anal., 31(3):915-930, 1994. MR 94m:65084

Department of Mathematics, Michigan State University, East Lansing, Michigan 48824-1027

E-mail address: li@mth.msu.edu

Department of Mathematics and Computer Science, University of Central Arkansas, Conway, Arkansas 72035-0001

E-mail address: wangx@cc1.uca.edu 